Supplement of The Cryosphere, 14, 4181-4199, 2020

https://doi.org/10.5194/tc-14-4181-2020-supplement

(C) Author(s) 2020. This work is distributed under

the Creative Commons Attribution 4.0 License.

(c) (1)

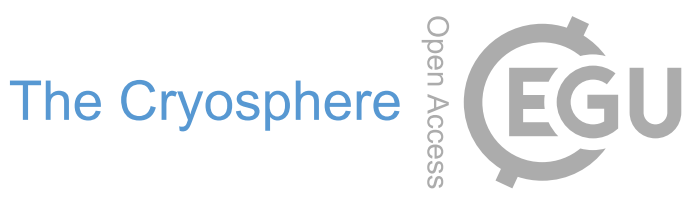

Supplement of

\title{
Long-term surface energy balance of the western Greenland Ice Sheet and the role of large-scale circulation variability
}

\section{Baojuan Huai et al.}

Correspondence to: Baojuan Huai (b.j.huai@uu.nl)

The copyright of individual parts of the supplement might differ from the CC BY 4.0 License. 


\section{Supplementary Materials}
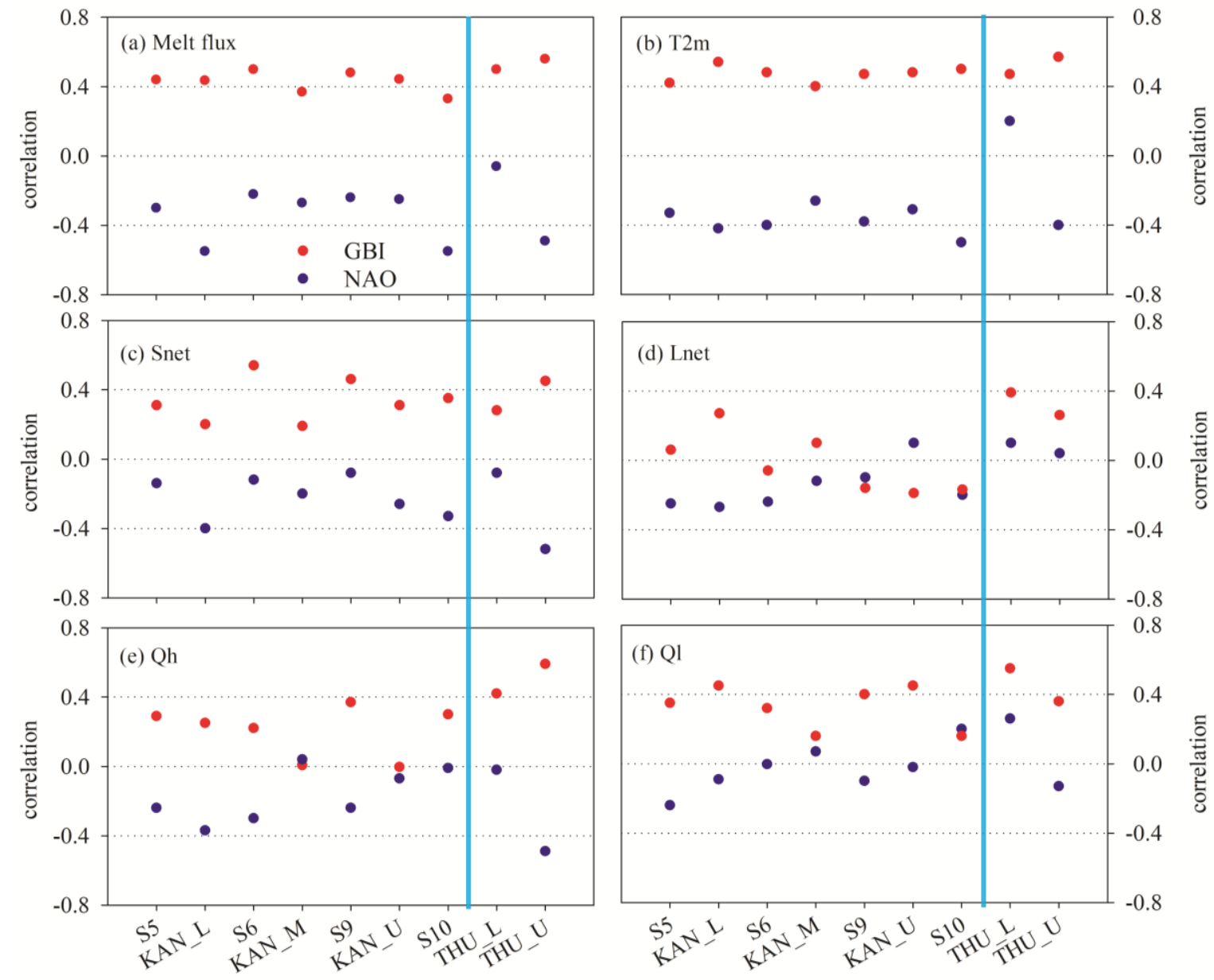

Figure S1. AWS correlations of JJA average SEB components and $2 \mathrm{~m}$ temperature (T2m) with GBI (red dots) and NAO index (blue dots). 

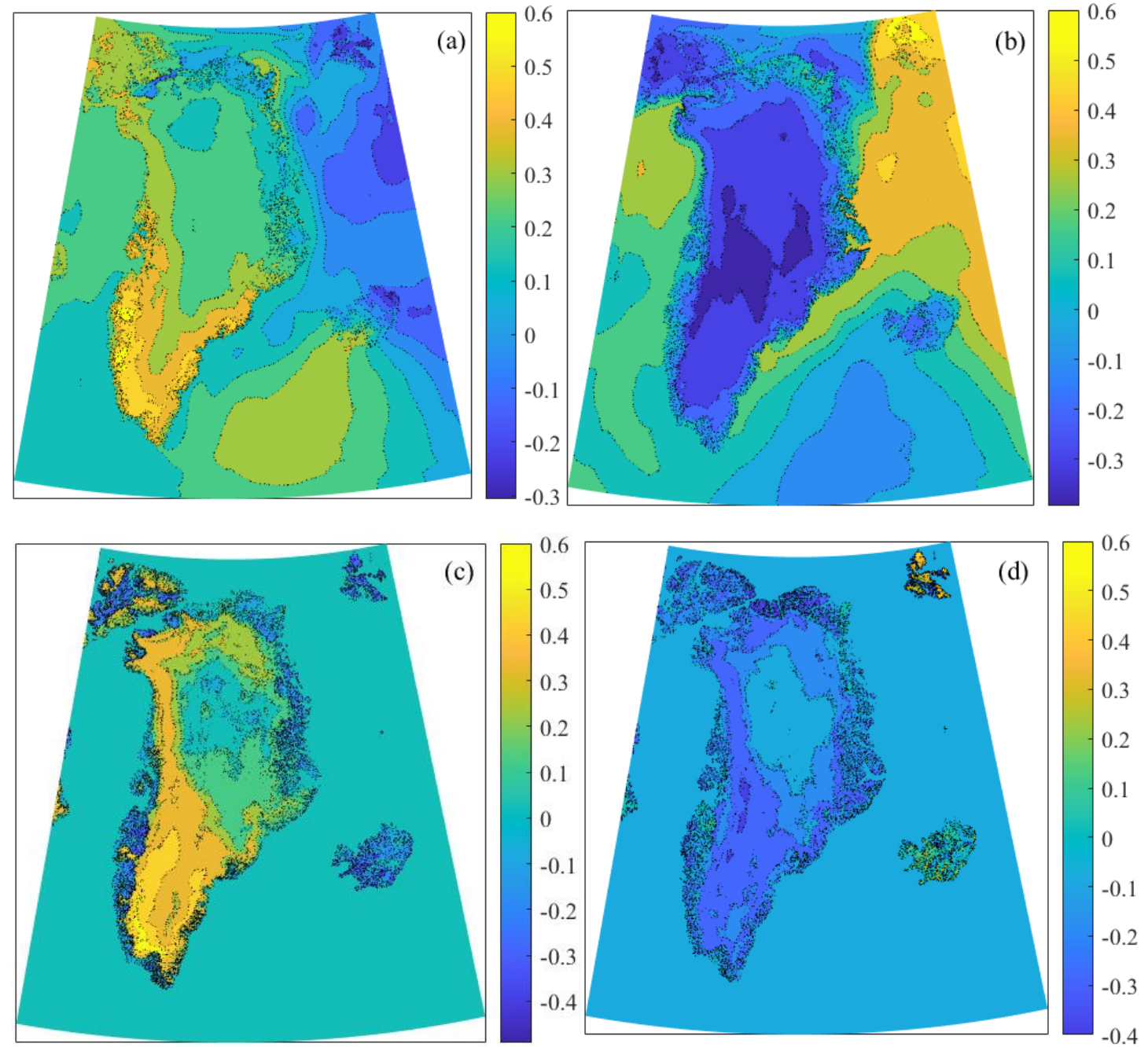

Figure S2. Correlation coefficient fields of 2000 2018 JJA average $2 \mathrm{~m}$ temperature (T2m) from RACMO2.3 with (a) GBI and (b) NAO, melt flux from RACMO2.3 with (c) GBI and (d) NAO index. 

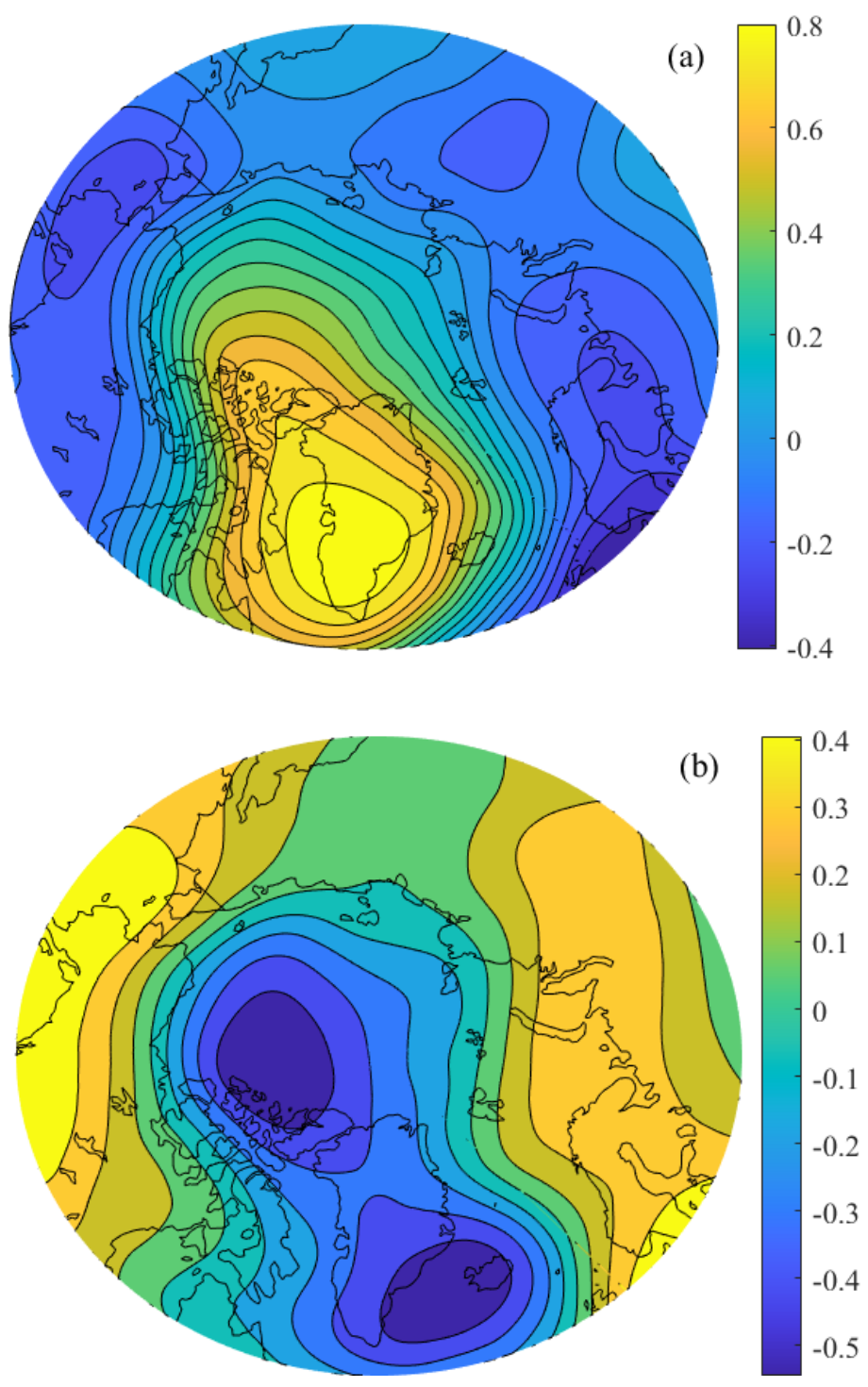

Figure S3. Regression fields of 2000 2018 JJA 500hpa geopotential height regressed with GBI (a) and NAO (b) index. The color bars show the correlation coefficient R. 
Table S1. Annual surface energy fluxes $\left(\mathrm{W} \mathrm{m}^{-2}\right)$ at the nine AWS locations, SEB values of $\mathrm{L}_{\mathrm{out}}, \mathrm{Q}_{\mathrm{h}}$, $\mathrm{Q}_{1}, \mathrm{G}$ and $\mathrm{M}$ are derived from the SEB model while $\mathrm{S}_{\mathrm{in}}, \mathrm{S}_{\mathrm{out}}$ and $\mathrm{L}_{\mathrm{in}}$ are from observations

\begin{tabular}{cccccccccc}
\hline Flux & S5 & KANL & S6 & KANM & S9 & KANU & S10 & THUL & THUU \\
\hline $\mathrm{S}_{\text {in }}$ & 124 & 129 & 146 & 141 & 146 & 146 & 155 & 108 & 113 \\
\hline $\mathrm{S}_{\text {out }}$ & -75 & -79 & -89 & -98 & -109 & -117 & -125 & -74 & -84 \\
\hline $\mathrm{S}_{\text {net }}$ & 49 & 50 & 57 & 43 & 37 & 29 & 31 & 34 & 29 \\
\hline $\mathrm{L}_{\text {in }}$ & 238 & 240 & 240 & 226 & 219 & 217 & 201 & 231 & 222 \\
$\mathrm{~L}_{\text {out }}$ & -277 & -276 & -274 & -263 & -258 & -250 & -237 & -264 & -256 \\
\hline $\mathrm{L}_{\text {net }}$ & -39 & -36 & -34 & -36 & -38 & -33 & -36 & -33 & -34 \\
\hline $\mathrm{R}_{\text {net }}$ & 10 & 14 & 23 & 6 & -1 & -4 & -6 & 2 & -5 \\
\hline $\mathrm{Q}_{\mathrm{h}}$ & 32 & 22 & 18 & 15 & 10 & 10 & 6 & 20 & 16 \\
\hline $\mathrm{Q}_{\mathrm{l}}$ & -4 & -6 & -2 & -5 & -1 & -4 & -1 & -6 & -2 \\
\hline $\mathrm{G}$ & -1 & 6 & -1 & 1 & 3 & 5 & 6 & 2 & 2 \\
\hline $\mathrm{M}$ & -37 & -36 & -39 & -17 & -11 & -6 & -5 & -17 & -11 \\
\hline
\end{tabular}

Table S2 Root Mean Squared Error (RMSE), mean bias (MB) and correlation coefficient (R) between daily AWS observations and ERA-Interim (EI), ERA5 (E5), RACMO2.3 (RAC) at KAN_L

\begin{tabular}{|c|c|c|c|c|c|c|c|c|c|c|c|c|c|}
\hline Variable & AWS & EI & E5 & RAC & \multicolumn{3}{|c|}{ EI } & \multicolumn{3}{|c|}{ E5 } & \multicolumn{3}{|c|}{ RAC } \\
\hline$S_{\text {out }}$ & 79 & 44 & 41 & 81 & 32 & 23.4 & 0.96 & 38 & 56 & 0.80 & 2 & 21 & 0.95 \\
\hline $\mathrm{Q}_{1}$ & -6 & -6 & -17 & -4 & 1.2 & 9 & 0.14 & 23 & 19 & 0.23 & 2 & 4 & 0.79 \\
\hline M & -36 & $\sim$ & $\sim$ & -38 & $\sim$ & $\sim$ & $\sim$ & $\sim$ & $\sim$ & $\sim$ & 1.6 & 12 & 0.97 \\
\hline $\operatorname{Alb}(-)$ & 0.70 & 0.64 & 0.74 & 0.74 & 0.1 & 0.27 & 0.5 & 0.06 & 0.2 & 0.6 & 0.02 & 0.13 & 0.78 \\
\hline
\end{tabular}

Table S3 Root Mean Squared Error (RMSE), mean bias (MB) and correlation coefficient (R) between daily AWS observations and ERA-Interim (EI), ERA5 (E5), RACMO2.3 (RAC) at KAN_M

\begin{tabular}{|c|c|c|c|c|c|c|c|c|c|c|c|c|c|}
\hline \multirow{2}{*}{ Variable } & \multirow{2}{*}{ AWS } & \multirow[t]{2}{*}{ EI } & \multirow{2}{*}{ E5 } & \multirow{2}{*}{ RAC } & \multicolumn{3}{|c|}{ EI } & \multicolumn{3}{|c|}{ E5 } & \multicolumn{3}{|c|}{ RAC } \\
\hline & & & & & MB & RMSE & $\mathrm{R}$ & MB & RMSE & $\mathrm{R}$ & MB & RMSE & $\mathrm{R}$ \\
\hline $\mathrm{S}_{\text {out }}$ & 98 & 78 & 118 & 99 & 15.4 & 34 & 0.93 & 20 & 44 & 0.92 & 1 & 11.5 & 0.99 \\
\hline $\mathrm{Q}_{1}$ & -5 & -6.6 & -7 & -4.5 & 1.6 & 9 & 0.5 & 2 & 8.9 & 0.4 & 0.5 & 3.6 & 0.85 \\
\hline M & -17 & $\sim$ & $\sim$ & -16.6 & $\sim$ & $\sim$ & $\sim$ & $\sim$ & $\sim$ & $\sim$ & 5.3 & 13 & 0.96 \\
\hline Alb (-) & 0.78 & 0.52 & 0.85 & 0.82 & 0.16 & 0.28 & 0.68 & 0.07 & 0.14 & 0.7 & 0.04 & 0.06 & 0.92 \\
\hline
\end{tabular}


Table S4 Root Mean Squared Error (RMSE), mean bias (MB) and correlation coefficient (R) between daily AWS observations and ERA-Interim (EI), ERA5 (E5), RACMO2.3 (RAC) at KAN_U

\begin{tabular}{|c|c|c|c|c|c|c|c|c|c|c|c|c|c|}
\hline \multirow{2}{*}{ Variable } & \multirow{2}{*}{ AWS } & \multirow[t]{2}{*}{ EI } & \multirow{2}{*}{ E5 } & \multirow{2}{*}{ RAC } & \multicolumn{3}{|c|}{ EI } & \multicolumn{3}{|c|}{ E5 } & \multicolumn{3}{|c|}{ RAC } \\
\hline & & & & & MB & RMSE & $\mathrm{R}$ & MB & RMSE & $\mathrm{R}$ & MB & RMSE & $\mathrm{R}$ \\
\hline $\mathrm{S}_{\text {out }}$ & 117 & 107 & 120 & 118 & 35.8 & 47 & 0.98 & 3 & 10 & 0.9 & 1 & 5 & 0.99 \\
\hline $\mathrm{Q}_{\mathrm{l}}$ & -4 & -6.9 & -3.1 & -4.5 & 2 & 3.3 & 0.95 & 1 & 5.4 & 0.75 & 0.5 & 4.6 & 0.78 \\
\hline M & -6 & $\sim$ & $\sim$ & -8 & $\sim$ & $\sim$ & $\sim$ & $\sim$ & $\sim$ & $\sim$ & 1.4 & 4.5 & 0.94 \\
\hline $\operatorname{Alb}(-)$ & 0.83 & 0.61 & 0.85 & 0.87 & 0.2 & 0.6 & 0.31 & 0.02 & 0.05 & 0.5 & 0.04 & 0.06 & 0.7 \\
\hline
\end{tabular}

Table S5 Root Mean Squared Error (RMSE), mean bias (MB) and correlation coefficient (R) between daily AWS observations and ERA-Interim (EI), ERA5 (E5), RACMO2.3 (RAC) at THU_L

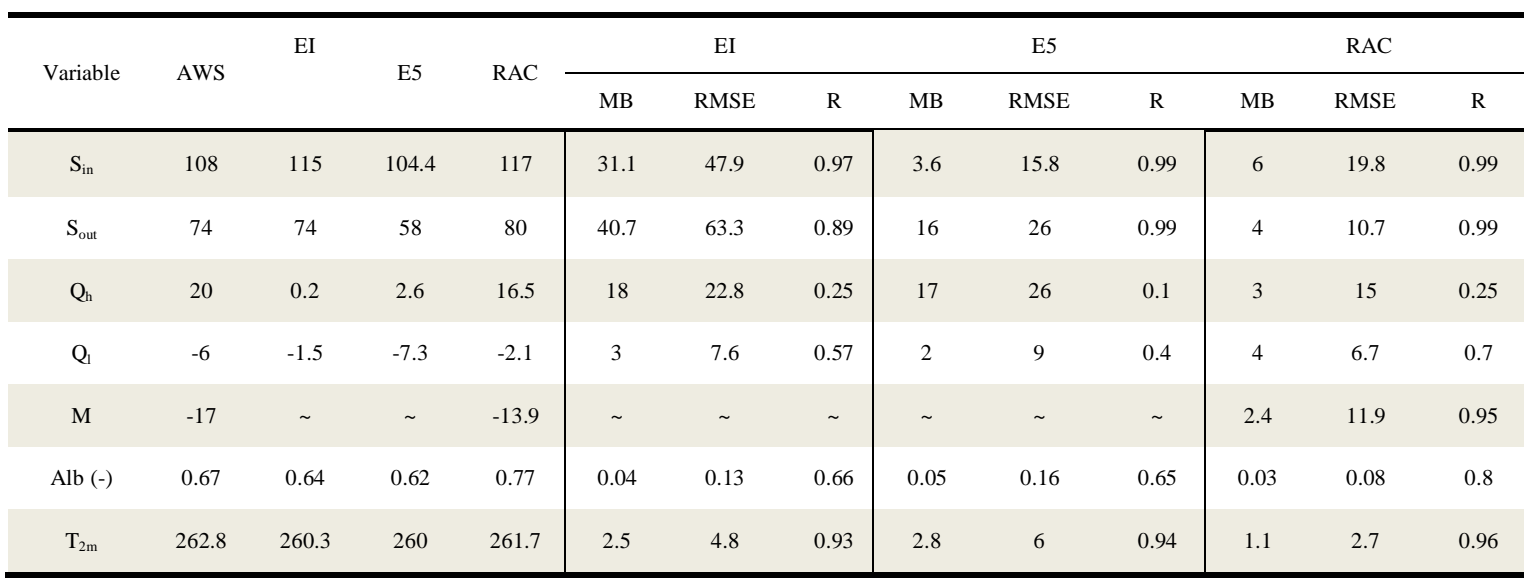

Table S6 Root Mean Squared Error (RMSE), mean bias (MB) and correlation coefficient (R) between daily AWS observations and ERA-Interim (EI), ERA5 (E5), RACMO2.3 (RAC) at THU_U

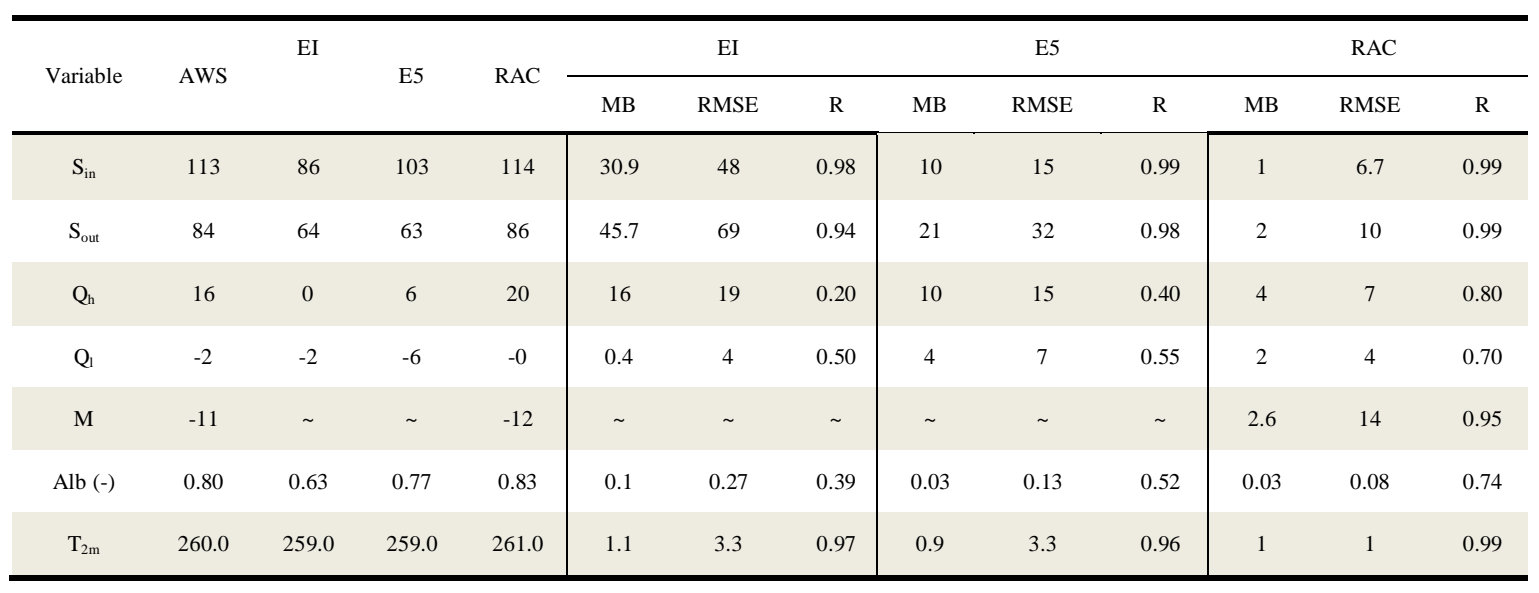

